\title{
lonic liquids: an emerging tool for an improved organic synthesis
}

\section{Editorial}

Ionic liquid is a class of organic salt comprise of organic cation and suitable inorganic or organic anion that remains in liquid state at ambient temperature. These ionic liquids have received a lot of attention of the scientific community due to their unusual and special properties such as low melting temperature, high thermal stability, large liquid state range, high solvation behavior towards organic or inorganic solutes, etc. ${ }^{1-4}$ The journey of ionic liquids was started with the development of ethyl ammonium nitrate as the first ionic liquid by Paul Walder in 1914. After that several classes of ionic liquids and their applications in the various areas have been reported. In 1999, the concept of designing ionic liquid to interact with a solute in a specific manner was demonstrated and introduced the term "task specific ionic liquid". 5

The diversity of possible combinations of organic cation and suitable inorganic or organic anion allows the design of ionic liquids suitable for the various organic reactions resulted in increased chemical yields, increased chemo-, regio-, and stereo selectivity as well as recycling of the catalyst. ${ }^{6}$ The covalent bond between organic cation/organic or inorganic anion of an ionic liquid and some functional group such as $\mathrm{NH}_{2}, \mathrm{OH}, \mathrm{SH}, \mathrm{OR}, \mathrm{PPh}_{2}, \mathrm{Si}(\mathrm{OR})_{3}$, urea and thiourea, metal complexes etc. impart the capacity to behave ionic liquid as an reagent, catalyst along with solvent characteristic in the chemical reactions. For example,

i. Bronsted acidic ionic liquids having sulphonic acid functional groups were used as a solvent and/or catalyst for esterification and other acid catalyzed reactions. ${ }^{7}$

ii. Ionic liquids having covalent bond with amines are able to separate carbon dioxide from gas streams. ${ }^{8}$

iii. Ionic liquids containing large aromatic head groups possess enhanced activity for extraction of aromatic compounds in aqueous biphasic systems. ${ }^{9}$

iv. Ionic liquids containing hydroxyl group $(-\mathrm{OH})$ have been utilized as phase transfer catalyst in the synthesis of ethoxybenzene. ${ }^{10}$

v. Ionic liquids containing metal lighting group have been utilized in the extraction of metal ions from aqueous solution. ${ }^{11}$

vi. Ionic liquids containing carboxylate groups have been used as supports for "Ionic Liquid phase" synthesis which is a versatile extension of the solid phase synthesis concept. ${ }^{12}$

The ionic liquids switching for conventional organic solvents is an emerging trend for the past decade to improve remarkable reaction selectivity, easy work-up by straightforward extraction, high yield isolation and purification, and efficient reusability. ${ }^{6}$ Recent chemical literature indicates that ionic liquids can be utilized as a solvent for almost any organic reaction. Earlier, ionic liquids were introduced as a green solvent but today their role is much extended beyond this boundary and frequently used as solvent or catalysts/reagents to control the organic reactions.
Volume I Issue 2 - 2017

\author{
Karan Singh \\ Department of Chemistry,Akal College of Basic Sciences, India
}

Correspondence: Karan Singh, Department of Chemistry,

Akal College of Basic Sciences, Eternal University, Baru Sahib, Sirmour- I73101, H.P, India, Tel +919882226019, Fax 91 1799276006, Email karansinghji@rediffmail.com

Received: April 06, 2017 | Published: April 26, 2017

The behavior of ionic liquids may be acidic, basic or organ catalyst depending upon the functional group attached either to the anion or cation or both of an ordinary ionic liquid. In view of the acidic behavior of ionic liquids, the replacement of harmful acids by reusable ionic liquids is one of the great catalytic systems in organic synthesis. The ionic liquids possessing acidic behavior were exploited as catalysts for many organic transformations like Koch carbonization, Pechmann reaction, Aza-Michael reaction, aldol condensation, esterification of alcohols, Beckmann rearrangement, oxidation reaction, Prins reaction and Mannich reactions to mention a few. ${ }^{5}$

The ionic liquids having basic functionalities are also of great interest as they showed higher catalytic efficiency than base catalyzed reactions. Further, ionic liquids can be used as soluble supports for catalyst/reagent immobilization. A number of organic reactions such as 1, 3-cycloadditions, Knoevengeal reaction, Suzuki coupling, oligosaccharide synthesis and so many were executed by using soluble ionic liquid support. ${ }^{6}$

Therefore, ionic liquids are not particularly used as solvents, they are presently used as catalyst and catalytic support in organic synthesis and their scope is beyond academic research laboratories to industry. To conclude, it may be said that there is much scope to explore the diverse applications of ionic liquids in the field of organic synthesis.

\section{Acknowledgements}

None.

\section{Conflict of interest}

The author declares no conflict of interest.

\section{References}

1. Qiao Y, Ma W, Theyssen N, et al. Temperature-Responsive Ionic Liquids: Fundamental Behaviors and Catalytic Applications. Chem Rev. 2017;117(10):6881-6928.

2. Dong K, Zhang S, Wang J. Understanding the Hydrogen Bonds in Ionic Liquids and Their Roles in Properties and Reactions. Chem Commun. 2016;52(41):6744-6764.

3. Podgorsek A, Jacquemin J, Padua AA, et al. Mixing Enthalpy for Binary Mixtures Containing Ionic Liquids. Chem Rev. 2016;116(10):60756106. 
4. Amarasekara AS. Acidic Ionic Liquids. Chem Rev. 2016;116:6133-6183.

5. Ratti R. Ionic Liquids: Synthesis and Applications in Catalysis. $A d v$ Chem. 2014;2014:16.

6. Sowmiah S, Cheng CI, Chu YH. Ionic Liquids for Green Organic Synthesis. Curr Org Synth. 2012;9(22):74-95.

7. Xing H, Wang T, Zhou Z, et al. Novel Brønsted-acidic ionic liquids for esterifications. Ind Eng Chem Res. 2005;44(11):4147-4150.

8. Bates ED, Mayton RD, Ntai I, et al. $\mathrm{CO}_{2}$ capture by a task-specific ionic liquid. J Am Chem Soc. 2002;124(6):926-927.

9. Visser AE, Holbrey JD, Rogers RD. Hydrophobic ionic liquids incorporating $\mathrm{N}$-alkylisoquinolinium cations and their utilization in liquid-liquid separations. Chem Commun (Camb). 2001;23:2484-2485.
10. Feng GR, Peng JJ, Qiu HY, et al. Synthesis of novel greener functionalized ionic liquids containing appended hydroxyl. Syn Comm 2007;37(16):2671-2675.

11. Visser AE, Swatloski RP, Reichert WM, et al. Task-specific ionic liquids for the extraction of metal ions from aqueous solutions. Chem Comm. 2001;1:135-136.

12. Fraga-Dubreuil J, Bazureau JP. Grafted ionic liquid phase-supported synthesis of small organic molecules. Tetrahedron Lett. 2001;42(35):60976100 . 